\title{
Design of Reclosing Device for Distribution Line in Small Hydropower Station
}

\author{
Chen Honghui \\ Guanshansi Road No. 2, Maoming, China \\ Maoming Power Supply Bureau, Guangdong POWER \\ GRID \\ Maoming China \\ ashe_11@126.com
}

\section{Su Xin}

Hongqi Road No. 278, Nankai District, Tianjin, China Chengxi Power Supply Bureau, Tianjin POWER GRID

Tianjin, China

susin@126.com

\author{
Fang Qiang \\ DaGu south Road No. 1310 Hexi District Tianjin China \\ Tianjin university of Technology and Education \\ Tianjin, China \\ ygenghuang@126.com
}

\author{
Zheng Wendong \\ DaGu south Road No. 1310 Hexi District Tianjin China \\ Tianjin Key Laboratory of Information Sensing and \\ Intelligent Control; \\ Tianjin, China \\ 1294891558@qq.com
}

\begin{abstract}
When the small hydropower stations are linked with the power system, it becomes difficult to judge the type of the fault which causes the running of the hydropower station unsteadily. If the fault takes place in the distribution line which links to the hydropower stations, the reclosing always fails. A new reclosing relay device based on programmable logic controller is designed to resolve the problem of the compatibility with the other relaying actions and to improve the success rate of reclosing. The simulation running of the device in Guangdong Province proves its effectiveness.
\end{abstract}

Keywords-Small hydropower station; Reclosing device; PLC; analog signal, Relay protection

\section{INTRODUCTION}

At present, small hydropower stations are always running parallel with the large power grid to stabilize the voltage and the frequency of the micro grid. However, when the fault in power system occurs, the relay protection device tends to fail to reclose due to the small hydropower stations instability in running. Also the difference of waterpower between dry season and wet season results in the output power fluctuation dramatically and it makes the relay device difficult to reclose[1].

When the fault takes place in the distribution line between the power system and the micro grid of small hydropower station, the relay device activates circuit breaker to isolate the micro grid from the power system. The relay device always fails to reclose because it is difficult to obtain the period when the voltage of the distribution line disappears or the time when the phase synchronization appear. The macro grid of small hydropower station is broke into an isolating island in which there is unbalance between the supply of power and the power consumption. When the frequency fluctuates beyond the threshold, the power supply is interrupted.
Even an instantaneous power interrupt results in a longtime accident[2-6].

Considering the effects of the instability in the running of small hydropower stations and the verification methods of the device, the protection device of reclosing function is always disable. This paper analyses the key technology of adaptive reclosing of distribution power line. A more effective adaptive reclosing device is designed to improve the success rate of reclosing, and to improve the reliability of power supply in small hydropower station[7-8].

\section{SYSTEM STRUCTURE}

As the main composition of the relay protection, the adaptive reclosing is the guarantee of the reliability of the power supply and the stable operation of power grid. Because of the characteristics of the power system and power distribution lines, PLC is select as the main controller. Fig .1 shows the process of automatic reclosing controlled by PLC. When PLC detects the signal of breaker's tripping operation, it processes logic calculation and then send out the instruction of reclosing [9-10].

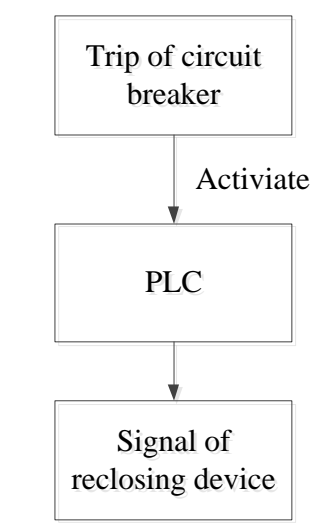

Figure 1. Process of PLC reclosing 
The reclosing device is designed based on modules, composing of relay protection function group and PLC component. Analog input is obtained by the analog subsystem. The signal is input by two ways: one is direct connection of the electric signal to PLC without signal conditioning and $\mathrm{AD}$ conversion, and the other is communication by PROFIBUS with a new digital device which samples the signal by signal conditioning and AD conversion.

When a fault takes place, the functional relay will act by the corresponding fault information. PLC samples the status of the relay and send them to the industrial personal computer by communication. The Industrial personal computer processes complicated calculation and send the result of the calculation back to PLC. PLC is the direct controller of the reclosing device[11-12]..

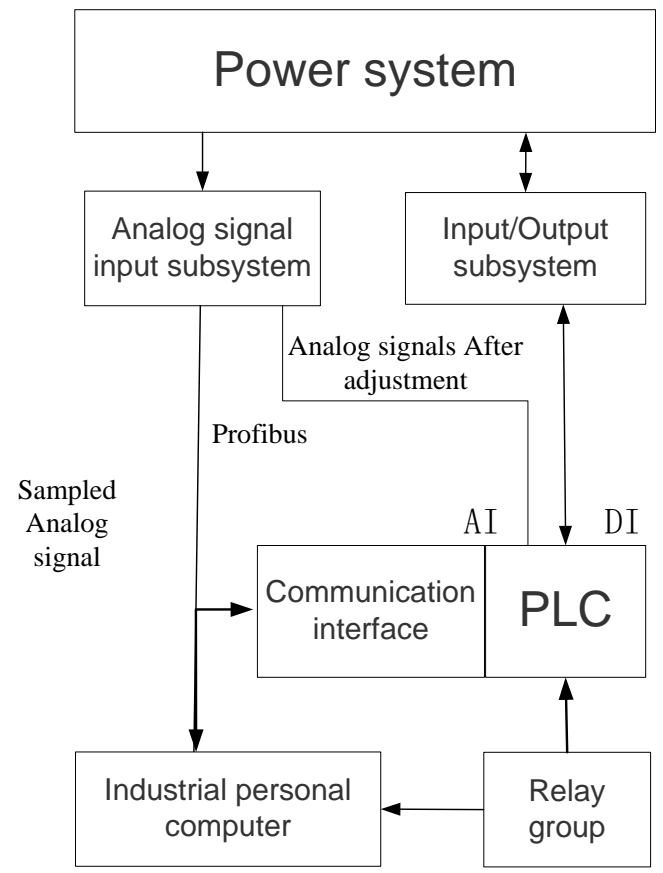

Figure 2. Flame of the device

\section{THE RECLOSING SYSTEM}

The reclosing device tests the changes of voltage, in order to make the process of the reclosing more simple, accurate, and reliable. Moreover, Artificial neural networks technology is used in this design, to improve the logical structure of the control system, allowing the devise to judge the fault type.

When a fault occurs in the circuit,line 2 will be cut. but the small hydropower station tends to work for more profits, only the frequency exceeds the specified upper or lower limit, the station will be cut from the system.

when reclosing device detects the fault in Transmission line is permanent and the frequency out of range, then cut the Generator5, the reclosing device do not act. However, when reclosing device detects the fault in Transmission line is Instantaneous fault, and he frequency out of range, then cut the Generator5.After that if the voltage become to $0 \mathrm{~V}$, then the reclosing device will close the Sectionalizer and the small hydropower station Connects to Power Grid.

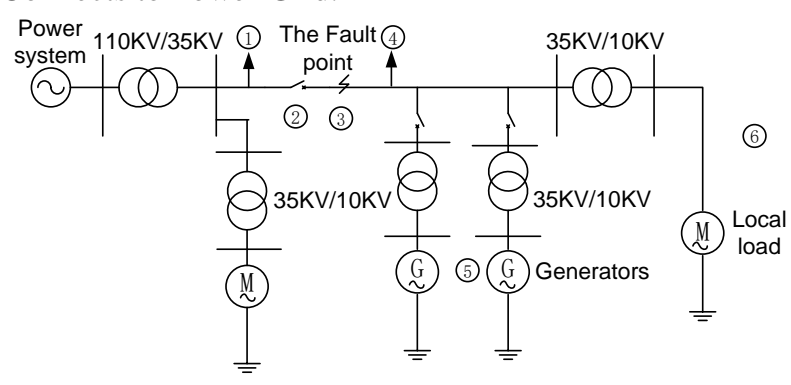

Figure 3. the reclosing system

\section{HARDWARE}

A. PLC

The using of PLC makes the action of reclosing device more accurate and reliable. According to the number of input/output (I/O) points and the speed requirement of the reclosing device, S7-300 of Siemens is selected. The model of PLC is CPU315-2PN/DP which includes internet and PROFIBUS-DP interface[13].

Fig . 3 shows the configure of I/O. Every I/O is used to link to one fault signal in order to detect the fault immediately.

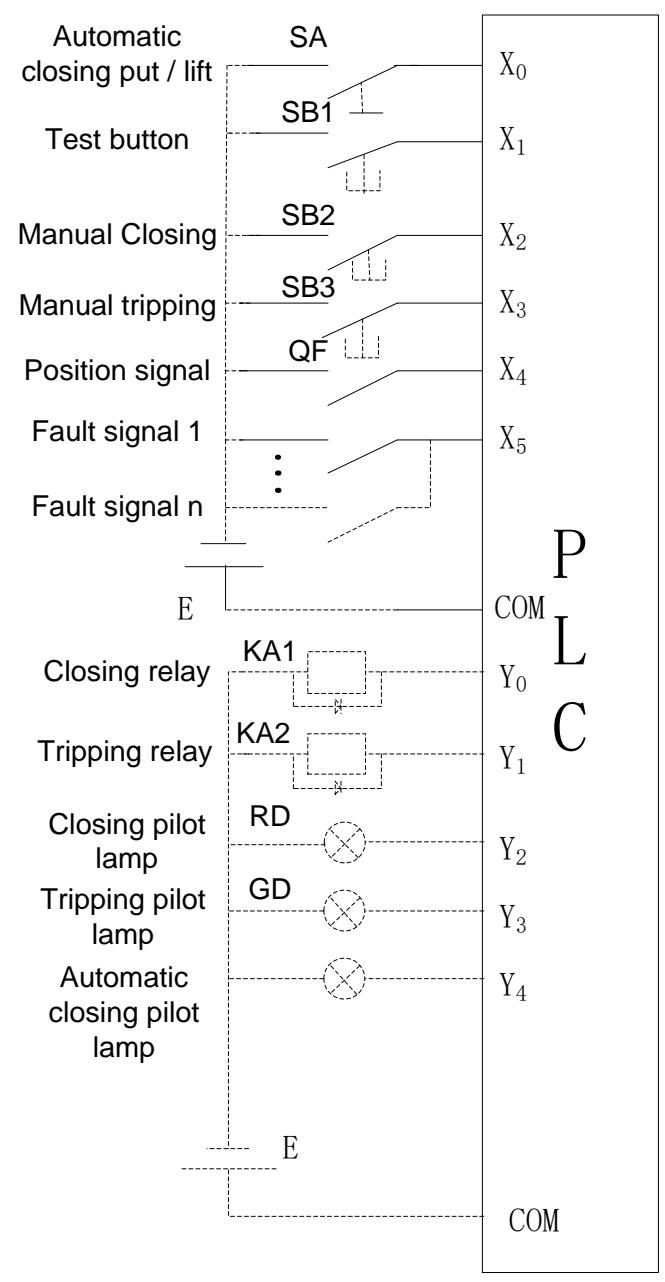

Figure 4. Configure of I/O 


\section{B. Analog signal sampling module}

As the voltage of signal from the TA and TV is too large to sample in the digital device, it is necessary to reduce the voltage to the range suitable for the digital measurement. Also the conditioning circuit of low pass filter is designed for the signal sampling. The voltage proportional to the voltage and current by TV and TA of the distribution line is obtained. The TA/TV conversion circuit, signal conditioning circuit and sampling circuit form the analog signal input module as Fig .4 shows.

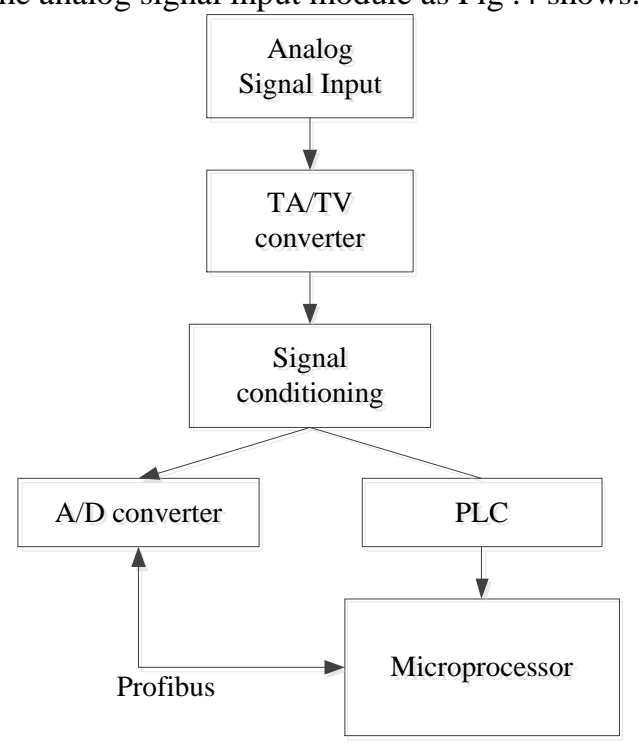

Figure 5. Process of the analog signal sampling

\section{Switch status detection and digital control}

The status of isolator, breaker and local or remote operation is the digital input of relay protection device. The breaker is also controlled by the digital signal. The switch status is obtained by PLC from input relay and photo-electricity isolator. The digital signal is transmitted after photo-electricity isolator and output relay. It is the most important for the reclosing device to design the detection circuit of the switch status because the correct status is the condition to act.

\section{Industrial personal computer}

Industrial personal computer is used to realize the mass data communication and complex calculation. Industrial personal computer communicates with PLC and the other digital device including relay protection device. Industrial personal computer obtains the operation information of relays, calculates the sampled analog signal by algorithm, and transmits the reclosing instruction to PLC.

\section{SOFTWARE DESIGN}

S7-300PLC is coded and compiled by STEP7 V5.5. The embedded internet interface is used to form the Ethernet and PROFIBUS networks. The process of the PLC control is shown by Fig .5.

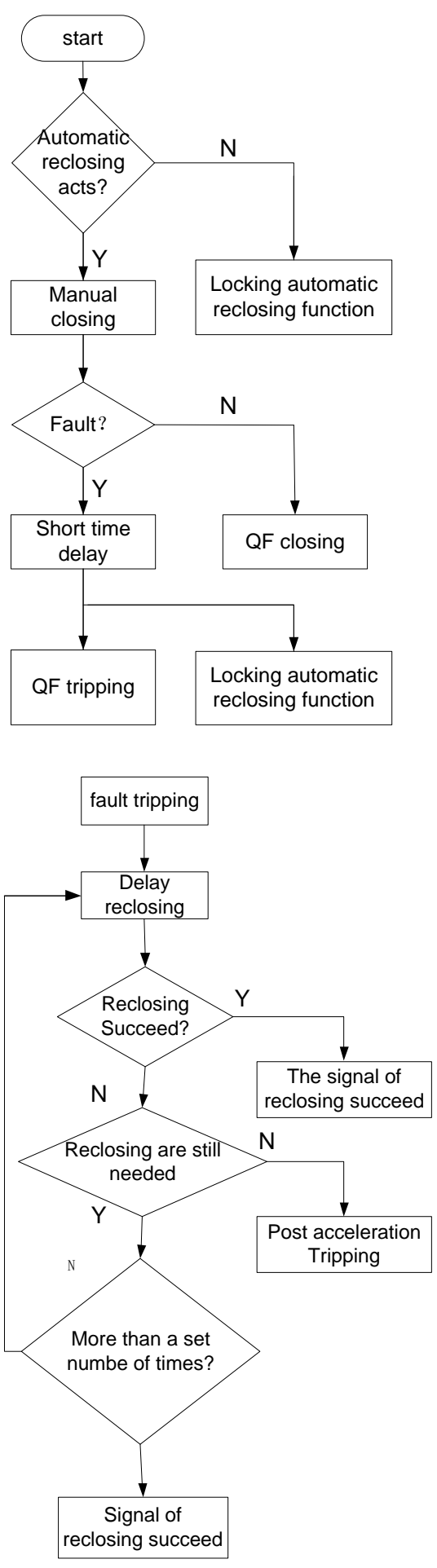

Figure 6. Process of the reclosing

\section{CONCLUSION}

The design of the reclosing device is based on the characteristics of the relay protection in power system which is connected to small hydropower station. The scheme is a collection of the PLC and industrial personal computer to improve the reliability of the reclosing device. Because of the reclosing control is based on more signal including both analog and digital, the device is more effective in reclosing. 


\section{ACKNOWLEDGMENT}

This work is supported by Guangdong POWER GRID Maoming Power Supply Bureau (K-GD2014-1023)

\section{REFERENCES}

[1] Platt G.,Cornforth D.,Berry A.Review of minigrid research and development around the world: accelerating the deployment of "Smart Minigrids"in APP countries. http://www. asiapacificpartnership. org /english/project_roster.aspx . 2009

[2] A.Griffo,D.Lauria."Optimal Reclosure Time for Improving Power System Dynamic Behaviour". IEE roc.-Gener . 2005

[3] Dugui Wu,,Zhiheng Xu.Development and prospect of microprocessor-based protection relays in China. Transmission and Distribution Conference and Exhibition 2002 . 2002

[4] Q. C. Le,B.T.T. Phan,T.Q.D. Khoa."Computation of Reclosing Time and Suitable Gain of Automatic VoltageRegulator". Power India Conference,2006, IEEE . 2006

[5] Negnevitsky M,,Pavlovsky V.Neural networks approach to online identification of multiple failures of protection systems. Power Delivery,IEEE Transactions on . 2005 R. Nicole, "Title of paper with only first word capitalized," J. Name Stand. Abbrev., in press.

[6] Li Cheng Sun,Zheng Wu. Relay Protection Based on Multi-Agent System[J]. Advanced Materials Research . 2013 (760)
[7] Guo Jia Rong,Ran Feng,Bi Zhuo,Xu Mei Hua. A Compiler for Ladder Diagram to Multi-Core Dataflow Architecture[J]. Advanced Materials Research . 2012 (462)

[8] Cunfeng Kang,Chong Wang,Chunmin Ma,Xudong Huang,Renyuan FEI. Run-time system based on LinSERCANS and Soft-PLC[J]. Frontiers of Mechanical Engineering in China 2009 (2)

[9] Zmaranda, Doina,Gabor, Gianina,Vancea, Codruta. Verification of PLC programs Used in Real-Time Applications[J]. Journal of Computer Science and Control Systems . 2010 (1)

[10] Gergely, Eugen Ioan,Husi, Geza,Yildirim, Sahin. PLC Programs Design Using Signal Interpreted Petri Networks[J]. Journal of Computer Science and Control Systems . 2009 (1)

[11] ]LasseterRobertH.CertsMicroghd[Cl.SystemofsystemsEngineering, 2007.SoSE.07.IEEEIntemationalConferenceon.SanAntonio,TX.20 07.

[12] Eto J.,Lasseter R.,Sehenkman B,et al.Overview of the Certs Microgrid LaboratoryTestBed=Cl.Integration of Wide-Seale Renewable Resources Into the Power Delivery System, 2009 CIGRE/IEEEPESJointSyn1Posium.Calgary,AB.2009:1-8.

[13] Mahat P.,Chen Zhe, Bak-Jensen B. Review of Islanding Deteetion Methods for Distributed Generation $=$ Cl.Electric Utility Deregulation and Restrueturing and Power Technologies, 2008 